\title{
Functionals of Stochastic Processes and Random Fields in Reliability Analysis
}

\author{
Claus Lange \\ Hochschule für Technik und Wirtschaft Dresden \\ Fachbereich Informatik/Mathematik \\ D-01069 Dresden, Friedrich-List-Platz 1 \\ Germany
}

\begin{abstract}
Probability theory methods for calculating approximations of first excursion and fatigue failure probabilities in the case of arbitrary differentiable stochastic process models are discussed. The discrete random variable of upcrossings of a level by a differentiable stochastic process is used to consider the functionals of stochastic processes which are of practical interest in reliability analysis.
\end{abstract}

\section{Introduction}

Many problems in reliability analysis of structural systems can be solved only by the theory of stochastic processes and random fields. Random excitations like wind, earthquake and sea waves, random loads like snow and vehicles, random temperature influences and random material or damping properties are only a few examples where methods of that theory are required to estimate the reliability of structural systems.

The approximation of first excursion or fatigue failure probabilities and of other characteristics of reliability bases on the distribution of different functionals of stochastic processes and random fields. The maximum and the minimum of a stochastic process, the distance between a local maximum and the following local minimum of a stochastic process, the time a process spends above or below a level and local maxima and minima of a random field are examples of such functionals.

It is given a method which approximates the distributions of all those functionals by the number of upcrossings (or downcrossings) of an arbitrary level by a stochastic process. The theory can be used to calculate the failure probability in the case where both types of failure for a structural system, that means fatigue failure as well as first excursion failure, are thinkable to occur. 


\section{First excursion failure models}

The reliability of structural systems is to determine in many engineering applications. It is assumed that the mathematical models of the structural systems, the random loads and the critical levels are given. The failure type is first excursion failure. An arbitrary differentiable (with probability one) stochastic process $X(t), t \in T \subset[0, \infty)$ is introduced for first excursion failure study. Typical functionals for reliability analysis are the random variables $X_{1}=\max X(t)$ or sometimes $X_{2}=\min X(t)$. Let be $S=\left[s_{A}, s_{E}\right] \subset T, c$ the critical level and $X\left(s_{A}\right)<c$ with probability one. In Lange [9] is discussed the approximation of probabilities

$$
P\left(\max _{t \in S} X(t)<c\right)=P(X(t)<c \forall t \epsilon S)
$$

by the discrete random variable $N$ of upcrossings of the level $c$ by $X(t), t \in T$ :

$$
P(N=0)=p_{0},
$$

especially with moments

$$
m_{i}=E N^{i}=\sum_{k=0}^{\infty} k^{i} p_{k}, m_{0}=1, i=1,2, \ldots ; P(N=k)=p_{k}
$$

and factorial moments

$$
m_{(i)}=E N(N-1) \ldots(N-i+1)=\sum_{k=0}^{\infty} k(k-1) \ldots(k-i+1) p_{k}, i=1,2, \ldots
$$

follow estimations like for example

$$
p_{0} \geq 1-m_{i}
$$

and series developments like

$$
p_{0}=1+\sum_{i=1}^{\infty} \frac{(-1)^{i}}{i !} m_{(i)}
$$

as well as Gram-Charlier-Series developments like

$$
p_{0}=e^{-m_{1}} \sum_{i=0}^{\infty} q_{i}(-1)^{i}
$$

with

$$
q_{0}=1, q_{1}=0, q_{i}=\sum_{j=0}^{i-1}(-1)^{j} \frac{m_{1}^{j}}{j !(i-j) !}\left(m_{(i-j)}-m_{1}^{i-j}\right), i=2,3, \ldots .
$$

The formulas (see Lange [9] ) are valid for arbitrary differentiable stochastic processes and arbitrary low and high constant levels, level functions and random levels, can be used for stochastic vector processes (see Engelund, Rackwitz, Lange [4] ) and can be generalized to random fields.

Here is the approximation of moments and factorial moments of the discrete random number $N$ very important (see for example Cramer, Leadbetter [3], Friedrich, Lange [5], Lange [9] ), especially basing on random counting measures. 


\section{Fatigue failure models}

Now the failure type is fatigue failure. A random measure $D(t)$ as a functional of the given arbitrary differentiable stochastic process $X(t), t \in T \subset[0, \infty)$ is introduced for modelling the fatigue in the following way:

$$
D(t)=\int_{0}^{t} G(D(\tau), S(\tau)) d \tau .
$$

$S(\tau)$ is the stress amplitude. $D(t)$ is normalized, that means at time $t=0$ is $D(t)=0$ with probability one and at time $t=L(L$ is the life time, a continuous random variable) is $D(L)=1$ with probability one. It is not necessary that $D(t)$ is a monotone increasing function.

Here is $G(\cdot, \cdot)$ a special integrable function depending on the practical problem ( for the deterministic case see Hennig, Friedrich, Heinrich, Mauersberger [8] ) with $G(D(\tau) ; S(\tau))>0$, if $S(\tau)>S_{D}\left(S_{D}\right.$ is the endurance limit) and $G(D(\tau), S(\tau))=0$, if $S(\tau) \leq S_{D}$. Formula (3.1) is a generalization for the deterministic Palmgren-Miner-Theory (see Bolotin [2] ; Heinrich, Hennig [7]). For the stochastic process $X(t), t \in T$ (which is now assumed to be a centralized narrowband process) the random measure $D(t)$ is given (in generalization from Heinrich, Hennig [7]) for example by

$$
D(t)=-\int_{0}^{t}\left[\ddot{X}(s) \delta(\dot{X}(s)) \chi\left(X(s)-S_{D}\right)\right] G(D(s), X(s)) d s .
$$

In $(3.2)$ are $\delta($.$) the Dirac delta distribution and$

$$
\chi\left(X(s)-S_{D}\right)=\left\{\begin{array}{l}
1 \text { if } X(t)>S_{D} \\
0 \text { if } X(t) \leq S_{D}
\end{array}\right.
$$

The calculation of the probability density function of the random variable $L$ from (3.2) and $D(L)=1$ is in general not possible. In the following the special case

$$
G(D(s), X(s))=\frac{1}{b_{2}}(X(s))^{b_{1}}=g(X(s))
$$

is considered, where $b_{1}, b_{2}$ are material constants. Let $E D(t)$ be the mean function of $D(t), t \epsilon[0, L]$ and $\sigma_{D}(t)$ the corresponding standard deviation function. The mean life time $E L$ can be approximated, if

$$
\lim _{t \rightarrow \infty} \frac{\sigma_{D}(t)}{E D(t)}=0
$$

can be shown, because in this case the probability that an arbitrary realization of $D(t)$ at time $t=E L=\tilde{L}$ is equal one, if $E D(\tilde{L})=1$, tends to one with increasing 
t. So the mean life time $\tilde{L}$ can be calculated from $E D(\tilde{L})=1$. Formulas $(3.2)$ and (3.3) give now

$$
E D(\tilde{L})=\int_{0}^{\tilde{L}}\left(\int_{S_{D}}^{\infty}\left(\int_{-\infty}^{0} \ddot{x} g(x) f(x, 0, \ddot{x}, t) d \ddot{x}\right) d x\right) d t=1,
$$

where $\mathrm{f}$ is the probability density function of the stochastic process $X(t), t \epsilon T$, its first and its second order derivatives. For stationary narrow band processes Renger, Mohr [12] proved the validity of (3.4). So further follows

$$
\tilde{L} \int_{S_{D}}^{\infty} \int_{-\infty}^{0} \ddot{x} g(x) f(x, 0, \ddot{x}) d \ddot{x} d x=\tilde{L} E D=1
$$

For a stationary narrow band Gaussian process $X(t), t \epsilon T$ follows from (3.6)

$$
E D=\frac{1}{b_{2}} \frac{1}{2 \pi} \frac{\sigma_{\dot{X}}}{\sigma_{X}}\left(\sqrt{2} \sigma_{X}\right)^{b_{1}} \Gamma\left(\frac{b_{1}+2}{2} ; \frac{1}{2}\left(\frac{S_{D}}{\sigma_{X}}\right)^{2}\right),
$$

with the gamma function

$$
\Gamma(x, y)=\int_{y}^{\infty} s^{x-1} \exp \{-s\} d s .
$$

A special case of (3.6) is the Miles formula (see Miles [11], Heinrich, Hennig [7]), which can be obtained with $S_{D}=0$ and $\Gamma(x, 0)=\Gamma(x)$ :

$$
E D=\frac{1}{b_{2}} \frac{1}{2 \pi} \frac{\sigma_{\dot{X}}}{\sigma_{X}}\left(\sqrt{2} \sigma_{X}\right)^{b_{1}} \Gamma\left(\frac{b_{1}+2}{2}\right) .
$$

\section{First excursion or fatigue failure}

In the following the theory is used to calculate the failure probability in the case where both types of failure for a structural system, that means fatigue failure as well as first excursion failure, are thinkable to occur. The probabilities of no first excursion failure

$$
P(X(t)<c \forall t \epsilon S)
$$

and of no fatigue failure

$$
P(D(t)<1 \forall t \epsilon S)
$$

were discussed in ch.2 and ch. 3 separately. If both types of failure are thinkable to occur, it has to be considered the probability

$$
P(\{D(t)<1\} \cap\{X(t)<c\} \forall t \epsilon S) .
$$

Functional $D(t), t \epsilon T$ and stochastic process $X(t), t \epsilon T$ are dependent. So for the calculation of (4.3) has to be used the conditional probability:

$$
P(\{X(t)<c\} \forall t \epsilon S) \cdot P(\{D(t)<1\} \mid\{X(t)<c\} \forall t \epsilon S) .
$$


The first factor in (4.4) is equal $P(N=0)$ corresponding ch.2. A new construction for the functional $D(t), t \epsilon S$ in generalization of Miles [11] and Heinrich, Hennig [7] is now derived to make possible the calculation of the second factor in (4.4):

Let $N_{c_{i}}\left(t_{1}, t_{2}\right)$ be the number of upcrossings of the level $c_{i}$ by $X(t), t \epsilon\left[t_{1}, t_{2}\right) \subset$ $T$ and $D_{c_{i}}\left(t_{1}, t_{2}\right)$ the growth of damage in an interval $\left[c_{i}, c_{i+1}\right)$ for $t \epsilon\left[t_{1}, t_{2}\right)$. It is clearly, that the growth of damage depends on the difference of the numbers of local maxima and local minima, the material function $g$ (see (3.3)) and the corresponding interval. The random variable "number of local maxima minus number of local minima in an interval $\left[c_{i}, c_{i+1}\right)$ " is equal $N_{c_{i}}\left(t_{1}, t_{2}\right)-N_{c_{i+1}}\left(t_{1}, t_{2}\right)$. Then is defined

$$
D_{c_{i}}\left(t_{1}, t_{2}\right)=\left[N_{c_{i}}\left(t_{1}, t_{2}\right)-N_{c_{i+1}}\left(t_{1}, t_{2}\right)\right] \cdot g\left(c_{i}\right)
$$

With $c_{1}=0$ and $c_{i+1}=c_{i}+\Delta, \Delta>0$ the damage $D\left(t_{1}, t_{2}\right)$ is now

$$
\begin{gathered}
D\left(t_{1}, t_{2}\right)=\sum_{i=1}^{\infty} D_{c_{i}}\left(t_{1}, t_{2}\right)=\sum_{i=1}^{\infty} g\left(c_{i}\right)\left[N_{c_{i}}\left(t_{1}, t_{2}\right)-N_{c_{i+1}}\left(t_{1}, t_{2}\right)\right] \\
=\sum_{i=1}^{\infty} N_{c_{i}}\left(t_{1}, t_{2}\right)\left[g\left(c_{i+1}\right)-g\left(c_{i}\right)\right]=\sum_{i=1}^{\infty} N_{c_{i}}\left(t_{1}, t_{2}\right)\left[\frac{g\left(c_{i}+\Delta\right)-g\left(c_{i}\right)}{\Delta}\right] \Delta .
\end{gathered}
$$

For $\Delta \rightarrow 0$ follows

$$
D\left(t_{1}, t_{2}\right)=\int_{0}^{\infty} N_{c}\left(t_{1}, t_{2}\right) \frac{\partial g(c)}{\partial c} d c .
$$

The formula for the mean value $E D\left(t_{1}, t_{2}\right)$ of the damage

$$
E D\left(t_{1}, t_{2}\right)=\int_{0}^{\infty} E N_{c}\left(t_{1}, t_{2}\right) \frac{\partial g(c)}{\partial c} d c
$$

is valid for an arbitrary differentiable stochastic process and by this formula can be approximated under certain simple assumptions the mean life time. Formula (4.7) is a generalization of the Miles formula.

Let $X(t), t \in T$ be a stationary Gaussian process with the probability density function $f$ of the stochastic process and its first order derivative:

$$
f(x, \dot{x} ; t)=\frac{1}{2 \pi \sigma_{X} \sigma_{\dot{X}}} \exp \left\{-\frac{x^{2}}{2 \sigma_{X}^{2}}\right\} \exp \left\{-\frac{\dot{x}^{2}}{2 \sigma_{\dot{X}}^{2}}\right\} .
$$

After the calculation of

$$
E N_{c}\left(t_{1}, t_{2}\right)=\frac{\left(t_{2}-t_{1}\right)}{2 \pi} \frac{\sigma_{\dot{X}}}{\sigma_{X}} \exp \left\{-\frac{1}{2}\left(\frac{c}{\sigma_{X}}\right)^{2}\right\}
$$

and with

$$
g(c)=\frac{1}{b_{2}} c^{b_{1}} ; \frac{\partial g(c)}{\partial c}=\frac{b_{1}}{b_{2}} c^{b_{1}-1}
$$


and (4.7) follows then

$$
\begin{gathered}
E D\left(t_{1}, t_{2}\right)=\frac{\left(t_{2}-t_{1}\right)}{2 \pi} \frac{\sigma_{\dot{X}}}{\sigma_{X}} \frac{b_{1}}{b_{2}} \int_{0}^{\infty} \exp \left\{-\frac{1}{2}\left(\frac{c}{\sigma_{X}}\right)^{2}\right\} c^{b_{1}-1} d c \\
=\ldots=\frac{\left(t_{2}-t_{1}\right)}{2 \pi b_{2}} \frac{\sigma_{\dot{X}}}{\sigma_{X}}\left(\sqrt{2} \sigma_{X}\right)^{b_{1}} \Gamma\left(\frac{b_{1}+2}{2}\right) .
\end{gathered}
$$

For nonstationary Gaussian processes can be obtained from (4.7) the mean

$$
E D\left(t_{1}, t_{2}\right)=\frac{1}{2 \pi b_{2}} \int_{t_{1}}^{t_{2}} \sqrt{1-\alpha^{2}(t)} \frac{\sigma_{\dot{X}}(t)}{\sigma_{X}^{2}(t)} I\left(\Theta, b_{1}, t\right) d t
$$

with

$$
\begin{gathered}
I\left(\Theta, b_{1}, t\right)=\int_{0}^{\infty} c^{b_{1}} \exp \left\{-\frac{1}{2}\left(\frac{c}{\sigma_{X}(t)}\right)^{2}\right\} \\
\cdot\left[\frac{c}{\sigma_{X}(t)} \exp \left\{-\frac{c^{2} \Theta^{2}}{2 \sigma_{X}^{2}(t)}\right\}-\sqrt{\frac{\pi}{2}} \Theta\left(1-\frac{c^{2}}{\sigma_{X}^{2}(t)}\right)\left(1+\Phi\left(\frac{c \Theta}{\sqrt{2} \sigma_{X}(t)}\right)\right)\right] d c \\
\alpha(t)=\frac{\rho_{X \dot{X}}(t)}{\sigma_{X}(t) \sigma_{\dot{X}}(t)} ; \Theta=\Theta(t)=\sqrt{\frac{\alpha^{2}(t)}{1-\alpha^{2}(t)}}
\end{gathered}
$$

and $\Phi(\cdot)$ as the (Gaussian) $N(0,1)$-distribution function.

Now the calculation of probability (4.3) will be considered. To simplify the notation is used without any restriction: $S=[0, t] ; t_{1}=0 ; t_{2}=t$ and $N_{c}(0, t)=$ $N_{c}(t) ; D(0, t)=D(t)$ :

$$
\begin{gathered}
p_{0}=p_{0}(t)=P\left(\{D(t)<1\} \cap\left\{N_{c}(t)=0\right\}\right) \\
=P\left(\left\{N_{c}(t)=0\right\}\right) \cdot P\left(\{D(t)<1\} \mid\left\{N_{c}(t)=0\right\}\right) .
\end{gathered}
$$

With (4.6) is then

$$
p_{0}(t)=P\left(\left\{N_{c}(t)=0\right\}\right) \cdot P\left(\left\{\int_{0}^{\infty} N_{c}(t) \frac{\partial g(c)}{\partial c} d c<1\right\} \mid\left\{N_{c}(t)=0\right\}\right)
$$

Formula (4.6) makes it possible to calculate the conditional probability in (4.9):

$$
p_{0}(t)=P\left(\left\{N_{c}(t)=0\right\}\right) \cdot P\left(\left\{\int_{0}^{c} N_{c}(t) \frac{\partial g(c)}{\partial c} d c<1\right\}\right) .
$$

The failure probability in the case where both types of failure for a structural system are thinkable to occur can be calculated on the basis of formula (4.10) for arbitrary differentiable stochastic processes. A lower bound for $p_{0}(t)$ is given by application of Markov inequality:

$$
p_{0}(t) \geq\left(1-E N_{c}(t)\right)\left(1-\int_{0}^{c} E N_{c}(t) \frac{\partial g(c)}{\partial c} d c\right)
$$


The distribution function for the discrete random variable $N=N_{c}(t)$ is approximately a Poisson distribution, if the critical level $c$ is "high enough" (see for example Cramer, Leadbetter [3] for Gaussian processes). So follows the lower bound

$$
p_{0}(t) \geq \exp \left\{-E N_{c}(t)\right\}\left(1-\int_{0}^{c} E N_{c}(t) \frac{\partial g(c)}{\partial c} d c\right)
$$

At last an example is considered. Let $X(t), t \in T$ be a stationary Gaussian process with mean function $E X(t) \equiv 0$, standard deviation function $\sigma_{X}(t) \equiv \sigma_{X}=1$ and standard deviation function of $\dot{X}(t), t \epsilon T$ (the derivative with probability one) $\sigma_{\dot{X}}(t) \equiv \sigma_{\dot{X}}$. Then it is easy to calculate

$$
p_{0}(t) \geq \exp \left\{-t \frac{\sigma_{\dot{X}}}{2 \pi} \cdot \exp \left[-\frac{c^{2}}{2}\right]\right\}\left(1-\frac{t \sigma_{\dot{X}}}{2 \pi} \frac{b_{1}}{b_{2}} \cdot 2^{\frac{b_{1}-2}{2}} \Gamma_{c}\left(\frac{b_{1}}{2}\right)\right)
$$

with the incomplete gamma function

$$
\Gamma_{c}(x)=\int_{0}^{c} y^{x-1} \exp \{-y\} d y .
$$

A generalization of the results for nonconstant critical levels is possible.

The following figure shows lower bounds for $p_{0}(t)$ corresponding formula (4.13) with $\sigma_{X}(t)=\sigma_{\dot{X}}(t) \equiv 1 ; b_{1}=2 ; b_{2}=40 ; c=3$ and $c=4$. It is easy to see, that the influence of a possible first excursion failure is small for high critical levels.

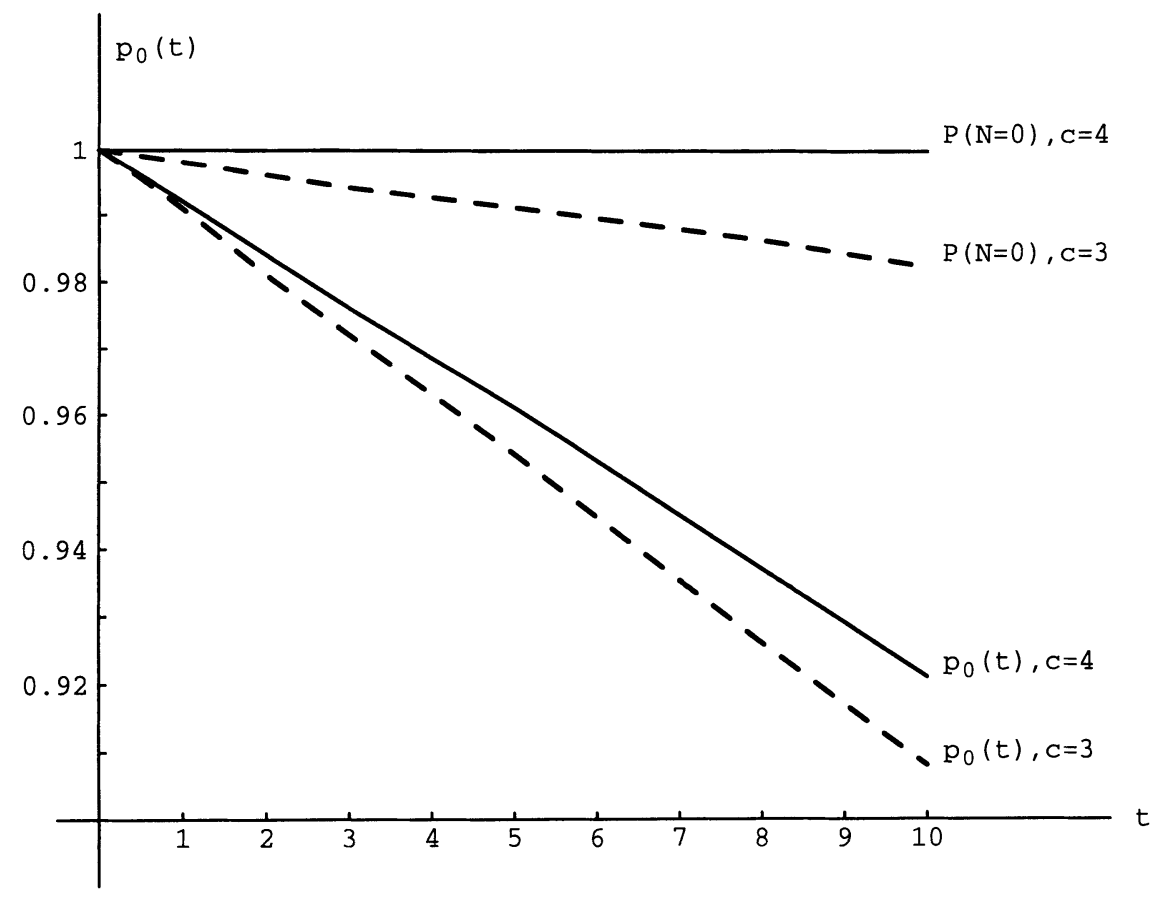




\section{References}

\section{[1] Beljajev,Y.K.}

Distribution of the maximum of a random field and its application to problems of reliability theory (in Russ.), Isv. Akad. Nauk SSSR, Techn. Kib. 2 (1970) 44-56

[2] Bolotin,V.V.

Wahrscheinlichkeitsmethoden zur Berechnung von Konstruktionen, Verlag für Bauwesen, Berlin 1981

[3] Cramer, H., Leadbetter, M.R.

Stationary and related processes, Wiley, New York 1969

[ 4] Engelund, S., Rackwitz, R., Lange, C.

Approximations of first-passage times for differentiable processes, publication in Prob. Eng. Mech. 1994

[ 5] Friedrich, H., Lange, C.

Niveauüberschreitungswahrscheinlichkeiten bei stochastisch belasteten mechanischen Systemen, Report R-Mech-04/81, Berlin 1981, 230 pp.

[ 6] Hasofer, A.M.

The mean number of maxima above high levels in Gaussian random fields, Journ. of Appl. Prob. 13 (1976) 377-379

[ 7] Heinrich, W., Hennig, K.

Zufallsschwingungen mechanischer Systeme, Akademieverlag, Berlin 1977

[ 8] Hennig, K., Friedrich, H., Heinrich, W., Mauersberger, G.

Studie zur Arbeit auf ausgewählten Gebieten der Sicherheit und Zuverlässigkeit im Maschinenbau und Bauwesen, AdW, ZIMM, Berlin 1979

[ 9] Lange, C.

First excursion probabilities for low threshold levels by differentiable processes, Proc. of the 4th WG 7.5 IFIP conference on reliability and optimization of structural systems 1991, Munich, Springer 1992, pp 261-275

[10] Lange, C., Friedrich, H.

Zuverlässigkeitsuntersuchungen für mechanische Systeme auf der Grundlage zufälliger Felder, FMC-Series No.43, Chemnitz 1989, 90 pp

[11] Miles, J.W.

On structural fatigue under random loading, Journal Aero. Sci. 21 (1954) 753-762

[12] Renger, A., Mohr, H.

Eine Studie über experimentielle Forschungen auf dem Gebiet der Zufallsschwingungen mechanischer Systeme, Forschungsbericht der HfR Festkörpermechanik, Berlin 1979 